\title{
Accuracy of FibroTouch in assessing liver steatosis and fibrosis in patients with metabolic-associated fatty liver disease combined with type 2 diabetes mellitus
}

\author{
Hongyan Yu ${ }^{1,2}$, Hui Liu ${ }^{2}$, Jie Zhang ${ }^{2}$, Guoyu Jia ${ }^{2}$, Ling Yang ${ }^{2}$, Qin Zhang ${ }^{2,3}$, Ge Li $^{4}$, Fang Liu ${ }^{2}$, \\ Fusheng Di $^{2}$, Fengmei Wang ${ }^{2,5}$
}

${ }^{1}$ Department of Endocrinology and Metabolism, The Third Central Clinical College of Tianjin Medical University, Tianjin, China; ${ }^{2}$ Tianjin Key Laboratory of Extracorporeal Life Support for Critical Diseases, Artificial Cell Engineering Technology Research Center, Tianjin Institute of Hepatobiliary Disease, The Third Central Hospital of Tianjin, Tianjin, China; ${ }^{3}$ Department of Pathology, Teda Hospital of Tianjin University, Tianjin, China; ${ }^{4}$ Public Health Science and Engineering College, Tianjin University of Traditional Chinese Medicine, Tianjin, China; ${ }^{5}$ Department of Gastroenterology and Hepatology, The Third Central Clinical College of Tianjin Medical University, Tianjin, China

Contributions: (I) Conception and design: F Wang, F Di; (II) Administrative support: F Wang, F Di; (III) Provision of study materials or patients: J Zhang, G Jia, L Yang; (IV) Collection and assembly of data: H Yu, Q Zhang, H Liu, F Liu; (V) Data analysis and interpretation: H Yu, G Li, F Wang; (VI) Manuscript writing: All authors; (VII) Final approval of manuscript: All authors.

Correspondence to: Fengmei Wang. Department of Gastroenterology and Hepatology, The Third Central Clinical College of Tianjin Medical University, Tianjin 300170, China. Email: wangfengmei@tmu.edu.cn; Fusheng Di. Department of Endocrinology and Metabolism, The Third Central Hospital of Tianjin, Tianjin 300170, China. Email: difusheng@vip.163.com.

Background Metabolic-associated fatty liver disease (MAFLD) is highly prevalent in type 2 diabetes mellitus (T2DM) patients and can rapidly progress to steatohepatitis, liver fibrosis, and hepatocellular carcinoma (HCC). Accurate evaluation and proper management of MAFLD can help prevent adverse liver outcomes. Here we evaluated the precision of the FibroTouch (FT) in the staging of liver steatosis and fibrosis in patients with MAFLD combined with T2DM using two indicators: controlled attenuation parameter (CAP) and liver stiffness measurement (LSM).

Methods: Eighty-five adult MAFLD combined with T2DM patients were selected at our center from July 2016 to July 2019 and underwent liver puncture biopsy for histopathology and the FT assay simultaneously. Two blinded pathologists independently reviewed the samples. The severity of fatty liver was classified using two scoring systems: the nonalcoholic fatty liver disease activity score (NAS) and the fibrosis score. Scores were then assessed following the Pathology Working Group of the NASH Clinical Research Network of the National Institutes of Health. Similarly, the severity of nonalcoholic steatohepatitis (NASH) was classified using the European Steatosis Activity Fibrosis (SAF) system. The FT assay was applied to obtain the LSM and the CAP. FT accuracy in diagnosing steatosis and fibrosis was determined by the receiver operating characteristic (ROC) curve and the area under the ROC curve (AUROC).

Results: Using biopsy analysis as the gold standard, the AUROCs and cutoff values of CAP in diagnosing liver steatosis were as follows: 0.84 (95\% CI: 0.67-1.01) and $278 \mathrm{~dB} / \mathrm{m}$ for S $\geq$ S1, 0.88 (95\% CI: 0.81-0.95) and $305 \mathrm{~dB} / \mathrm{m}$ for $\mathrm{S} \geq \mathrm{S} 2,0.89$ (95\% CI: 0.82-0.95) and $307 \mathrm{~dB} / \mathrm{m}$ for $\mathrm{S} \geq \mathrm{S} 3$. The AUROCs and cutoff values of LSM in diagnosing liver fibrosis were as follows: 0.76 (95\% CI: 0.66-0.86) for F $\geq \mathrm{F} 2,0.81$ (95\% CI: $0.71-0.91$ ) and $13.8 \mathrm{kPa}$ for $\mathrm{F} \geq \mathrm{F} 3,0.92$ (95\% CI: 0.85-1.00) and $20.1 \mathrm{kPa}$ for F $\geq \mathrm{F} 4$.

Conclusions: In patients of MAFLD with T2DM, CAP and LSM obtained by FT are highly accurate in assess liver steatosis and fibrosis, respectively, with AUROC values ranging from 0.76 to 0.92 .

Keywords: FibroTouch (FT); liver biopsy; controlled attenuation parameter (CAP); liver stiffness measurements (LSMs) 
Submitted Jul 08, 2021. Accepted for publication Sep 10, 2021.

doi: 10.21037/apm-21-2339

View this article at: https://dx.doi.org/10.21037/apm-21-2339

\section{Introduction}

Fatty liver disease (FLD) has become the leading chronic liver disease in the 21 st century, with a current global prevalence of about $25 \%$, posing a serious threat to human health and raising a huge socioeconomic burden (1-3). To increase the detection rate of FLD and enable early intervention and treatment to delay complications, an international expert consensus 2020 proposed a new concept of metabolic-associated fatty liver disease (MAFLD). The diagnosis of MAFLD does not require the exclusion of alcoholic FLD or other chronic liver diseases, but requires the presence of metabolic abnormalities (2). Hepatic steatosis alone represents the early stage of MAFLD and can be reversed. However, it will progress to steatohepatitis, cirrhosis, and even hepatocellular carcinoma (HCC) without early intervention or if other metabolic-related diseases coexist (4). Studies have found that $12-44 \%$ of patients with hepatic steatosis progressed to steatohepatitis, and $15 \%$ of steatohepatitis progressed to cirrhosis or HCC $(5,6)$. As one of aspect of a multi-system disease, MAFLD can not only cause adverse liver outcomes, but also cause other metabolic related complications: its most important complication is cardiovascular disease (CVD), followed by cancer, obstructive sleep apnea, polycystic ovarian syndrome, chronic kidney disease (CKD) and osteoporosis (3).

Epidemiological studies have shown a strong bidirectional relationship between MAFLD and T2DM. T2DM is strongly correlated with steatohepatitis progression to steatohepatitis, liver fibrosis, and/or HCC, MAFLD is associated with an increased risk of macrovascular and microvascular complications of T2DM and chronic kidney diseases (7-9). In patients with T2DM, the prevalence rates of MAFLD and steatohepatitis ranged $70-95 \%$ and $17.6-22 \%$, respectively $(5,10)$. The prevalence of steatohepatitis in patients with diabetes who underwent liver biopsy was as high as $64 \%$, and the prevalence of advanced liver fibrosis (F3) was $10.4 \%$ (11). The severity of hepatic fibrosis is the most powerful determinant of longterm outcomes, including mortality (12). As the incidences of steatohepatitis and liver fibrosis are significantly higher in T2DM patients, grading liver fibrosis in T2DM patients with MAFLD is crucial.
Although liver biopsy is the gold standard for diagnosing hepatic steatosis and staging of fibrosis, it is an invasive examination of the liver, which is usually difficult for most patients. Furthermore, sampling errors and unsuitability for long-term repeated treatment follow-up limit its clinical use. Therefore, the search for a noninvasive diagnostic method for the early identification of MAFLD and its associated liver fibrosis has been a hot research topic in recent years. Many novel techniques for the noninvasive assessment of liver fibrosis have been available, among which transient elastography (TE) is the most widely used in clinical practice. Its vibration-controlled TE measures the velocity of mechanical shear waves through the liver. The measured stiffness value of the liver is used as a marker of liver fibrosis, and the measured fat attenuation value of the liver is a marker of liver steatosis. The value of FibroScan for assessing the degree of liver fibrosis has been validated in different types of liver disease and is included in many expert consensuses and guidelines (13-15). FibroTouch (FT) is the first liver stiffness-elasticity detector in China, developed with the participation of Tsinghua University in 2010. Integrating two-dimensional (2D) imaging and TE techniques use built-in 2D ultrasound to guide accurate localization and detect liver tissue morphology, degree of liver fibrosis and steatosis. FT uses a broadband probe that emits a wider ultrasound band $(2-7 \mathrm{MHz})$ with both high-frequency and low-frequency components, which can adjust the penetration and resolution according to different examinees and thus improves the success rate and accuracy of the examinations. Thus, FT is especially valuable for obese people. The efficacy of FT in staging steatosis and fibrosis in patients with chronic liver disease has been well documented (16-19). In patients of MAFLD with T2DM, by using FibroScan, the threshold values of CAP for steatosis and the LSM for fibrosis have been variably reported. Although FT has more advantages than FibroScan in obese populations, no study with liver biopsy findings as the gold standard has investigated the diagnostic thresholds of FT in grading steatosis and fibrosis in MAFLD patients with T2DM.

Here we designed a prospective diagnostic study to evaluate the efficacies of FT-measured controlled attenuation parameter (CAP) and liver stiffness measurement in the 
staging of liver steatosis and fibrosis in MAFLD patients with T2DM, and to determine the corresponding cutoff values, aiming to provide a scientific basis for clinical noninvasive diagnosis and response assessment of this patient population. We present the following article in accordance with the STARD reporting checklist (available at https:// dx.doi.org/10.21037/apm-21-2339).

\section{Methods}

\section{Subjects and design}

In this prospective study, with liver biopsy results for staging liver steatosis and liver fibrosis as the gold standard, FT examination was conducted concurrently. Independent, blinded examiners compared the findings to assess the precision of the CAP and the liver stiffness measurement (LSM) in the diagnosis of liver steatosis and fibrosis. 85 patients with MAFLD combined with T2DM were recruited at the Third Central Hospital of Tianjin from July 2016 to July 2019. Liver histopathology was performed, and a FT examination was performed within 1 week before or after biopsy. The diagnosis of T2DM followed the WHO criteria for 1999, and the diagnosis of fatty liver was based on the Guidelines for the Management of Nonalcoholic Fatty Liver Disease (2010 Revision) (20). The Ethics Committee of the Third Central Hospital of Tianjin approved this study (IRB2016-006-02). This study was conducted in accordance with the Declaration of Helsinki (as revised in 2013) and Good Clinical Practice (GCP) for drugs. All subjects signed informed consents.

Exclusion criteria included: (I) aged $<18$ years; (II) alcohol consumption: more than $30 \mathrm{~g} / \mathrm{d}$ for men and $20 \mathrm{~g} / \mathrm{d}$ for women; (III) with chronic liver diseases including viral hepatitis, autoimmune hepatitis, HCC, decompensated liver disease, hepatolenticular degeneration, and/or drugrelated hepatitis; (IV) with diseases/conditions affecting glycolipid metabolism, including total parenteral nutrition, inflammatory bowel disease, anterior hypopituitarism, hyperthyroidism, Cushing syndrome, hemochromatosis; (V) using drugs that can cause fatty liver, including glucocorticoids, synthetic estrogens, olanzapine; (VI) with type 1 diabetes, gestational diabetes, or special types of diabetes; (VII) with acute complications of diabetes, severe infections, end-stage renal disease, blood disease and/or other complications due to metabolic disorder; (VIII) had participated in another clinical trial within the last 30 days.

\section{General data}

The following general information was collected: age, sex, height, weight, calculated BMI, and medical history (diabetes, hypertension, and hyperlipidemia). Fasting blood samples were collected for routine blood tests and determination of liver function, kidney function, lipids, ferritin, C-reactive protein, insulin, C-peptide, and blood glucose using an automatic biochemistry analyzer (Hitachi 7060: Tokyo, Japan) at the Third Central Hospital of Tianjin, and the test kits were purchased from Roche (Germany). Glycated hemoglobin (HbA1c) was measured with high-performance liquid chromatography using an automatic HbA1c analyzer (Toshiba G8L: Tokyo, Japan) at the Third Central Hospital of Tianjin.

\section{Pathological evaluation}

Ultrasound-guided liver biopsy was performed. Liver tissue samples were obtained using a MAXCORE 16G disposable automatic biopsy needle (Bard, USA) and fixed in $4 \%$ neutral formaldehyde. Each liver tissue sample was $1.6 \mathrm{~cm}$ in length, $1.2-1.8 \mathrm{~mm}$ in width, and contained more than 11 portal tracts. The samples were routinely dehydrated, paraffin embedded, and serially sectioned $4 \mu \mathrm{m}$ thick. The sections were stained with hematoxylin and eosin (H\&E), Masson \& E trichrome stain, and Gordon-Sweets reticular fiber stain. Two blinded pathologists independently reviewed the samples. The severity of nonalcoholic steatohepatitis (NASH) was classified using the European Steatosis Activity Fibrosis (SAF) system: steatosis (0-3), hepatocellular ballooning (0-2), lobular inflammation (0-3), and fibrosis (0-4) (21). Meanwhile, the NAFLD activity score (NAS) developed by the Pathology Working Group of the NASH Clinical Research Network of the National Institutes of Health was used (22). The typical pathological findings of different stages of liver steatosis and fibrosis are shown in Figure 1.

\section{Measurement of liver stiffness and controlled attenuation parameter with FT}

According to the manufacturer's instructions, FT (Wuxi Hisky Medical Technology Co. Ltd., Wuxi, Jiangsu, China) was used to measure liver stiffness measurement and controlled attenuation parameter by the same skilled operator using a blinding approach. The subjects were asked to take a supine position during the measurements, with the 

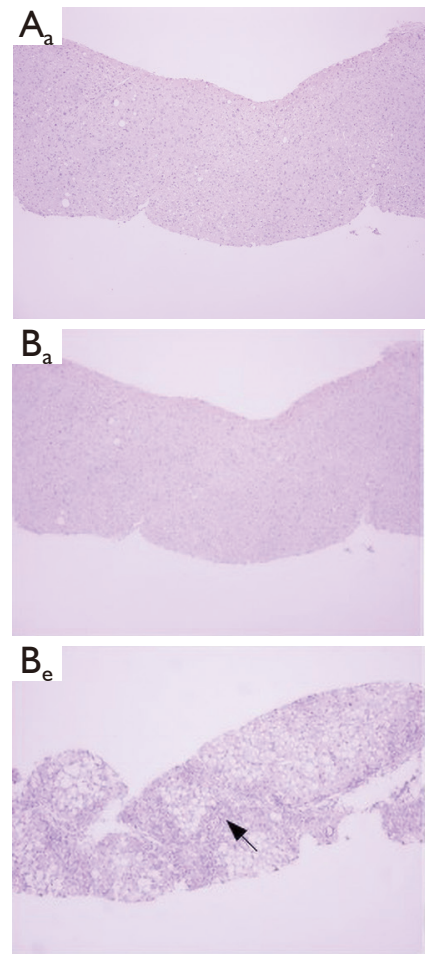
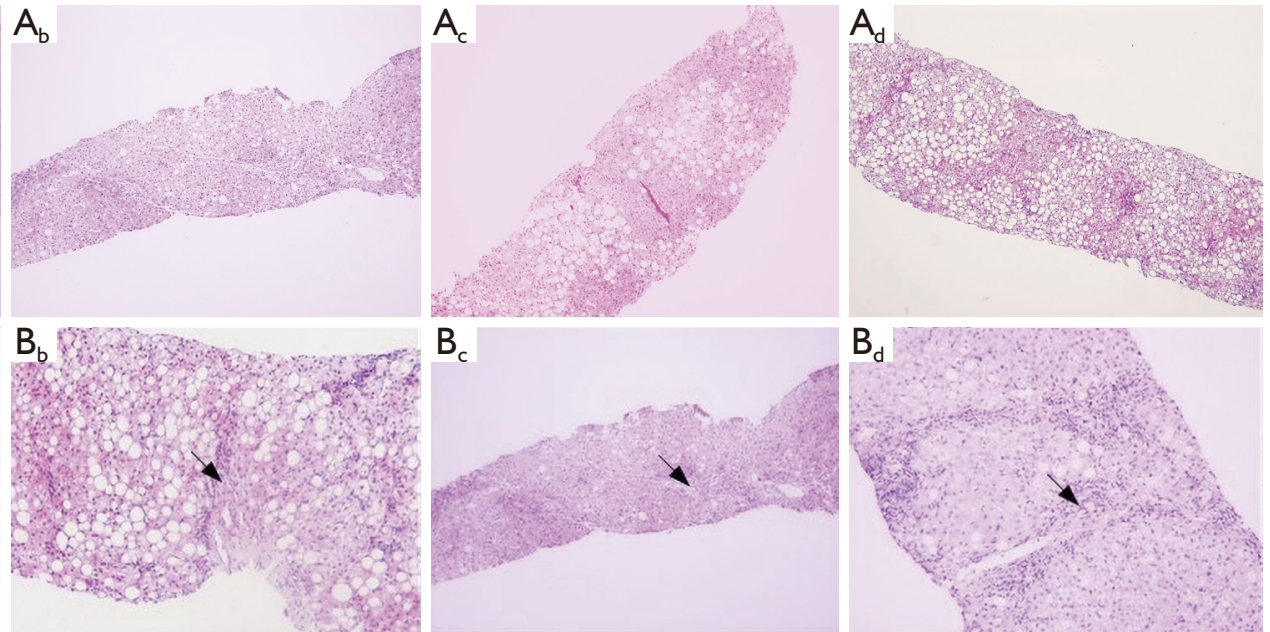

$\mathrm{B}_{\mathrm{c}}$
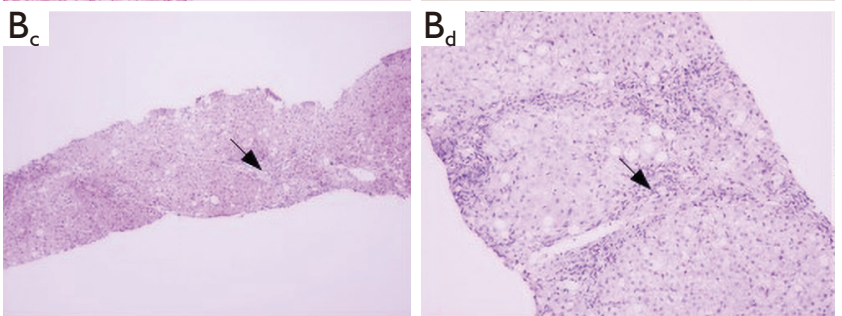

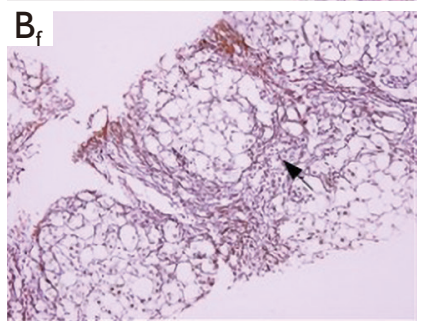

Figure 1 The typical pathological findings of different stages of hepatic steatosis and fibrosis. (A) The pathological stage of hepatic steatosis: a: S0 (\% of hepatocytes containing visible macrovascular steatosis <5\%); b: S1 (5-33\%); c: S2 (34-66\%); d: S3 (>66\%). HE ×100. (B) The pathological stage of liver fibrosis: a: F0 (no fibrosis); b: F1 (1a, mild perisinusoidal fibrosis or periportal fibrosis in hepatic alveolar zone 3; 1b, moderate perisinusoidal fibrosis in hepatic alveolar zone 3; and 1c, periportal fibrosis); c: F2 (perisinusoidal fibrosis in alveolar zone 3 combined with periportal fibrosis); d: F3 (bridging fibrosis); e: F4 (highly suspicious or confirmed cirrhosis); and f: reticular fiber staining of stage F4. B: (a, c, and e): HE, $\times 100$; (b,d): HE, $\times 200$; (f): reticular fiber staining, $\times 200$. The black arrows indicate the location of liver fibrosis.

right hand holding the head to obtain a maximal extension of the intercostal space. First, the FT ultrasound probe was placed between the 7th, 8th, and 9th ribs from the right anterior axillary line to the midaxillary line, avoiding cysts, blood vessels, and other structures in liver tissue that could interfere with the precision of the examination. After the position and angle of the examination were decided, the probe was moved to the test interface for measurement, during which the probe was kept perpendicular to the skin surface in the intercostal space. Ten successful examinations at the same site were considered valid (19).

\section{Statistical analysis}

Continuous variables are expressed as medians and interquartile ranges (IQRs), and categorical variables are expressed as percentages, for which $95 \%$ confidence intervals (CIs) were calculated. The Kruskal-Wallis test was used to compare the differences between the groups in steatosis classification and fibrosis classification with CAP and LSM. The Mann-Whitney U test was used to compare the differences between the medians. The correlations between CAP and pathological stage of steatosis, LSM and pathological stage of fibrosis were analyzed using Spearman rank correlation analysis. AUROC and the $95 \%$ confidence interval were used to estimate the diagnostic precision of CAP and LSM. The maximum Youden index (i.e., sensitivity + specificity - 1), sensitivity $\geq 90 \%$ and specificity $\geq 90 \%$ were used to determine the optimal cut-off values of CAP and LSM for the diagnosis of steatosis and fibrosis, and the sensitivity (SEN), specificity (SPE), positive predictive value (PPV), negative predictive value (NPV), positive likelihood ratio (PLR) and negative likelihood ratio (NLR) of each value and their corresponding $95 \%$ confidence intervals were calculated. Statistical analysis was performed using the SPSS 21.0 software package, and the curves were constructed 


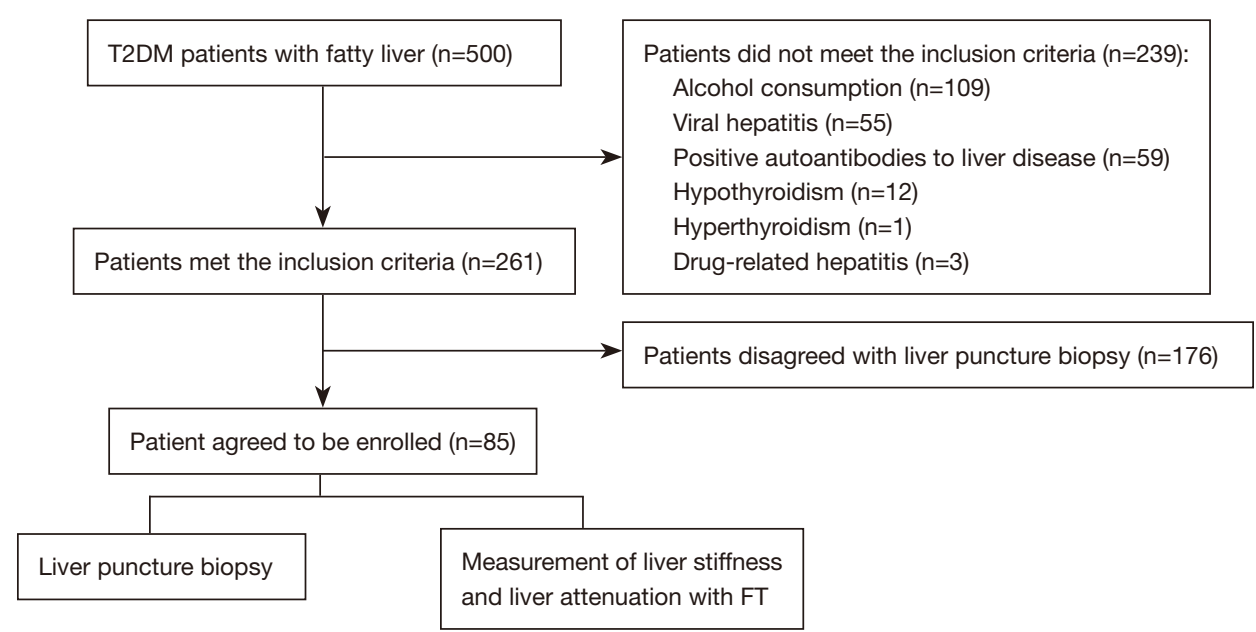

Figure 2 Research flow chart. Of the 500 patients with fatty liver combined with T2DM, 261 met the enrollment criteria and 85 underwent a liver puncture biopsy and a FibroTouch examination.

with the GraphPad Prism 5.0 software. A P value $<0.05$ was considered significantly different.

\section{Results}

\section{General information of subjects}

Figure 2 is the research flow chart. In this study, 85 patients [including 51 females (60.0\%) and 34 males (40.0\%)] aged 24-74 years (median: 58 years) were included. According to the SAF diagnostic criteria, 10 (11.8\%) cases with NAFL were diagnosed, 35 (41.2\%) with early NASH (F0-F1), 34 $(40.0 \%)$ with fibrotic NASH (F2-F3) and 7 (8.2\%) with cirrhosis (Table 1).

\section{Correlation between FT measurements and liver pathological findings}

CAP was positively correlated with the degree of steatosis ( $r=0.726$; 95\% CI: 0.662-0.804: $\mathrm{P}<0.01)$. LSM was positively correlated with the degree of liver fibrosis (r=0.537; 95\% CI: 0.372-0.678: $\mathrm{P}<0.01$ ).

\section{Distribution of CAP in patients with different degrees of steatosis}

The median CAP was $264 \mathrm{~dB} / \mathrm{m}$ (IQR, 209-295 dB/m) in group S0, $282 \mathrm{~dB} / \mathrm{m}(\mathrm{IQR}, 265-299 \mathrm{~dB} / \mathrm{m})$ in group $\mathrm{S} 1$, $306 \mathrm{~dB} / \mathrm{m}(\mathrm{IQR}, 291-331 \mathrm{~dB} / \mathrm{m})$ in group $\mathrm{S} 2$ and $341 \mathrm{~dB} / \mathrm{m}$ (IQR, 317-355 dB/m) in group S3. CAP increased with the degree of steatosis, and the comparison between four groups showed a significant difference $(\mathrm{P}<0.01)$; although the difference between groups S0 and S1 did not show a significant difference $(\mathrm{P}>0.05)$, the results of other pairwise comparisons were statistically significant (all $\mathrm{P}<0.05)$ (Figure $3 A$ ).

\section{Distribution of LSM in patients with different degrees of liver fibrosis}

The median LSM was $9.9 \mathrm{kPa}(\mathrm{IQR}, 7.7-11.4 \mathrm{kPa})$ in group F0, $11.1 \mathrm{kPa}(\mathrm{IQR}, 8.8-12.9 \mathrm{kPa})$ in group $\mathrm{F} 1,13.2 \mathrm{kPa}$ (IQR, 8.6-18.5 kPa) in group F2, $14.3 \mathrm{kPa}$ (IQR, 11.4$20.2 \mathrm{kPa}$ ) in group $\mathrm{F} 3$, and $24.1 \mathrm{kPa}(\mathrm{IQR}, 20.1-33.9 \mathrm{kPa})$ in group F4. LSM increased with the degree of liver fibrosis, and the comparison between five groups showed significant difference $(\mathrm{P}<0.0001)$. Pairwise comparisons showed significant differences between group F0 and group F4, between group F1 and group F3, and between group F2 and group $\mathrm{F} 4$ (all $\mathrm{P}<0.05$ ), whereas the remaining results were not significantly different (all $\mathrm{P}>0.05$ ) (Figure $3 B$ ).

\section{Cutoff values of CAP for differentiating different degrees of steatosis}

According to histopathological analysis, the distribution of different degrees of steatosis was as follows: S0 in 5 cases (5.9\%), S1 in 24 cases (28.2\%), S2 in 29 cases (34.1\%) and $\mathrm{S} 3$ in 27 cases $(31.8 \%)$. The optimal cutoff value of CAP in diagnosing $\mathrm{S} \geq \mathrm{S} 1$ was $278 \mathrm{~dB} / \mathrm{m}$, and the CAP value was $270 \mathrm{~dB} / \mathrm{m}$ for sensitivity $\geq 90 \%$, and $313 \mathrm{~dB} / \mathrm{m}$ for specificity 
Table 1 Patient characteristics

\begin{tabular}{|c|c|c|}
\hline Characteristics & Distribution & Range \\
\hline Age (years) & $58[20]$ & $24-74$ \\
\hline Female gender, n (\%) & $51(60.0)$ & - \\
\hline Height (cm) & $165[16]$ & $148-193$ \\
\hline Weight (kg) & $84[28]$ & $55-140$ \\
\hline $\mathrm{BMI}\left(\mathrm{kg} / \mathrm{m}^{2}\right)$ & $29.7(6.2)$ & $22.8-42.3$ \\
\hline Waist (cm) & $103[15]$ & $71-132$ \\
\hline Hipline (cm) & 106 [13] & $85-140$ \\
\hline WHR & $0.97(0.07)$ & $0.66-1.08$ \\
\hline HR (bpm) & $78[11]$ & $52-104$ \\
\hline Systolic pressure (mmHg) & $142[20]$ & $108-214$ \\
\hline Diastolic pressure $(\mathrm{mmHg})$ & $80[18]$ & $55-118$ \\
\hline Hypertension, n (\%) & $55(64.7)$ & - \\
\hline ASCVD, n (\%) & $37(43.5)$ & - \\
\hline Platelets count $\left(\times 10^{9} / \mathrm{L}\right)$ & $217[88]$ & 53-359 \\
\hline INR & $0.95(0.1)$ & $0.8-1.13$ \\
\hline AST (IU/L) & $23(23.5)$ & $4-151$ \\
\hline ALT (IU/L) & $47(46.5)$ & $5-217$ \\
\hline GGT (IU/L) & $48(32.5)$ & $13-367$ \\
\hline ALP (IU/L) & $82[51]$ & $15-189$ \\
\hline Albumin (g/L) & $64.8(6.2)$ & $53.5-84.6$ \\
\hline TBIL $(\mu \mathrm{mol} / \mathrm{L})$ & $11.9(6.65)$ & $3.8-29.3$ \\
\hline DBIL $(\mu \mathrm{mol} / \mathrm{L})$ & $4.4(2.50)$ & $3-13$ \\
\hline IBIL $(\mu \mathrm{mol} / \mathrm{L})$ & $7.50(4.35)$ & $2.6-18.8$ \\
\hline $\mathrm{HbA1c}(\%)$ & $9.0(2.8)$ & $5.2-15.1$ \\
\hline Fasting glucose (mmol/L) & $6.9(3.21)$ & $3.57-15.84$ \\
\hline Posting glucose (mmol/L) & $16.63(6.22)$ & $6.46-27.5$ \\
\hline Total cholesterol (mmol/L) & $4.5(1.45)$ & $2.60-8.87$ \\
\hline HDL cholesterol (mmol/L) & $0.97(0.33)$ & $0.46-2.61$ \\
\hline LDL cholesterol (mmol/L) & $2.56(1.25)$ & $1.07-5.34$ \\
\hline Triglyceride (mmol/L) & $1.99(1.17)$ & $0.87-24.22$ \\
\hline Ferritin (ng/mL) & $244.00(226.48)$ & $17.5-1,097.4$ \\
\hline Urea (mmol/L) & $4.89(1.60)$ & $2.32-22.52$ \\
\hline
\end{tabular}

Table 1 (continued)
Table 1 (continued)

\begin{tabular}{|c|c|c|}
\hline Characteristics & Distribution & Range \\
\hline Creatinine $(\mu \mathrm{mol} / \mathrm{L})$ & 62 [19] & $37-127$ \\
\hline hsCRP $(\mu \mathrm{g} / \mathrm{mL})$ & $3.62(5.87)$ & $0.24-17.10$ \\
\hline $\mathrm{ESR}(\mathrm{mm} / \mathrm{hr})$ & $13[12]$ & $3-83$ \\
\hline Uric acid ( $\mu \mathrm{mol} / \mathrm{L})$ & 307 (122.5) & $167-600$ \\
\hline $\mathrm{mACR}(\mathrm{mg} / \mathrm{mmol})$ & $0.84(3.20)$ & $0.07-64.92$ \\
\hline LSM (kPa) & $12.2(7.7)$ & $5.3-36.0$ \\
\hline $\mathrm{CAP}(\mathrm{dB} / \mathrm{m})$ & $312[49]$ & $188-400$ \\
\hline \multicolumn{3}{|l|}{ Fibrosis stage, n (\%) } \\
\hline Fo & $4(4.7)$ & - \\
\hline $\mathrm{F} 1$ & $37(43.5)$ & - \\
\hline $\mathrm{F} 2$ & $19(22.4)$ & - \\
\hline F3 & $18(21.2)$ & - \\
\hline F4 & $7(8.2)$ & - \\
\hline \multicolumn{3}{|c|}{ Steatosis grade, n (\%) } \\
\hline so & $5(5.9)$ & - \\
\hline S1 & $24(28.2)$ & - \\
\hline $\mathrm{S} 2$ & $29(34.1)$ & - \\
\hline S3 & $27(31.8)$ & - \\
\hline \multicolumn{3}{|c|}{ Ballooning grade, $\mathrm{n}(\%)$} \\
\hline BO & $6(7.1)$ & - \\
\hline B1 & $35(41.2)$ & - \\
\hline B2 & $44(51.8)$ & - \\
\hline \multicolumn{3}{|c|}{$\begin{array}{l}\text { Lobular inflammation grade, } \mathrm{n} \\
\text { (\%) }\end{array}$} \\
\hline 10 & $2(2.4)$ & - \\
\hline 11 & $19(22.4)$ & - \\
\hline 12 & $38(44.7)$ & - \\
\hline 13 & $26(30.6)$ & - \\
\hline \multicolumn{3}{|l|}{ NAS Score, n (\%) } \\
\hline $0-2$ & $5(5.9)$ & - \\
\hline $3-4$ & $19(22.4)$ & - \\
\hline $5-8$ & $61(71.8)$ & - \\
\hline
\end{tabular}

Table 1 (continued) 
Table 1 (continued)

\begin{tabular}{lcc}
\hline Characteristics & Distribution & Range \\
\hline Activity grade (according to SAF), $\mathrm{n}(\%)$ & \\
A0 & $1(1.2)$ & - \\
A1 & $2(2.4)$ & - \\
A2 & $12(14.1)$ & - \\
A3 & $34(40.0)$ & - \\
A4 & $36(42.4)$ & - \\
\hline
\end{tabular}

The data are shown as figure (percentage) or median (interquartile range). BMI, body mass index; WHR, waist-hip ratio; HR, heart rate; ASCVD, atherosclerotic cardiovascular disease; INR, international normalized ratio; AST, aspartate aminotransferase, ALT, alanine transaminase, GGT, gammaglutamyl transferase; ALP, alkaline phosphatase; TBIL, total bilirubin; DBIL, direct bilirubin; IBIL: indirect bilirubin; HDL, highdensity lipoprotein; LDL, low-density lipoprotein; hsCRP, highsensitivity c-reactive protein; ESR, erythrocyte sedimentation rate; $\mathrm{mACR}$, microalbumin/creatinine ratio; LSM, liver stiffness measurement; CAP, controlled attenuation parameter; NAS, NAFLD activity score.

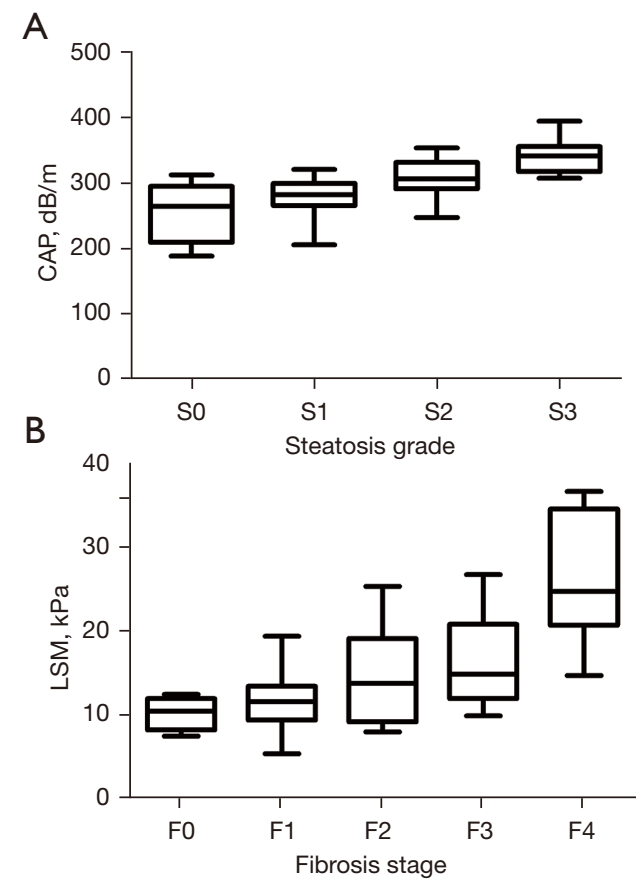

Figure 3 Box plots of various pathological stages of liver steatosis and fibrosis. (A) Box plot of CAP and pathological stages of liver steatosis; (B) Box plot of LSM and pathological stages of liver fibrosis. CAP, controlled attenuation parameter; LSM, liver stiffness measurement. $\geq 90 \%$. The optimal cutoff value of CAP in diagnosing $\mathrm{S} \geq \mathrm{S} 2$ was $305 \mathrm{~dB} / \mathrm{m}$, and the CAP value was $286 \mathrm{~dB} / \mathrm{m}$ for sensitivity $\geq 90 \%$, and $312 \mathrm{~dB} / \mathrm{m}$ for specificity $\geq 90 \%$. The optimal cutoff value of CAP in diagnosing $\mathrm{S}=\mathrm{S} 3$ was $307 \mathrm{~dB} / \mathrm{m}$, and the CAP value was $309 \mathrm{~dB} / \mathrm{m}$ for sensitivity $\geq 90 \%$, and $335 \mathrm{~dB} / \mathrm{m}$ for specificity $\geq 90 \%$ (Table 2 ).

\section{LSM cutoff values to differentiate different degrees of liver fibrosis}

According to the histopathological analysis, the distribution of different degrees of liver fibrosis was as follows: F0 in 4 cases $(4.7 \%), \mathrm{F} 1$ in 37 cases $(43.5 \%), \mathrm{F} 2$ in 19 cases (22.4\%), $\mathrm{F} 3$ in 18 cases $(21.2 \%)$ and $\mathrm{F} 4$ in 7 cases $(8.2 \%)$. The optimal cutoff value of LSM in diagnosing $\mathrm{F} \geq \mathrm{F} 2$ was $12.8 \mathrm{kPa}$, and the LSM was $8.5 \mathrm{kPa}$ for sensitivity $\geq 90 \%$ and $15.1 \mathrm{kPa}$ for specificity $\geq 90 \%$; The optimal cutoff value of $\mathrm{LSM}$ in diagnosing $\mathrm{F} \geq \mathrm{F} 3$ was $13.8 \mathrm{kPa}$, and the $\mathrm{LSM}$ was $10.6 \mathrm{kPa}$ for sensitivity $\geq 90 \%$ and $18.3 \mathrm{kPa}$ for specificity $\geq 90 \%$; The optimal cutoff value of LSM in diagnosing $\mathrm{F}=$ F4 was $20.1 \mathrm{kPa}$, and the LSM was $14.1 \mathrm{kPa}$ for sensitivity $\geq 90 \%$ and $20.1 \mathrm{kPa}$ for specificity $\geq 90 \%$ (Table 3 ).

\section{ROC curves and AUROC of CAP in differentiating different degrees of steatosis}

The AUROC of CAP was 0.84 (0.67-1.01) for $\mathrm{S} \geq \mathrm{S} 1,0.88$ $(0.81-0.95)$ for $\mathrm{S} \geq \mathrm{S} 2,0.89(0.82-0.95)$ for $\mathrm{S}=\mathrm{S} 3$ (Figure 4).

\section{ROC curves and LSM AUROC in differentiating different degrees of liver fibrosis}

The AUROC of LSM was $0.76(0.66-0.86)$ for $\mathrm{F} \geq \mathrm{F} 2$, $0.81(0.71-0.91)$ for $F \geq F 3$, and $0.92(0.85-1.00)$ for $F=F 4$ (Figure 5).

\section{Discussion}

In MAFLD patients $(2,23)$, simple hepatic steatosis itself is a benign and reversible lesion. However, liver steatosis will progress to steatohepatitis, liver fibrosis, or even cirrhosis and HCC when it persists for a long time, especially in the coexistence of hypertension and dyslipidemia, insulin resistance, sleep apnea, and other risk factors (1). MAFLD and T2DM are causal factors, and the progression of NAFLD to NASH, liver fibrosis, and HCC will be accelerated in the presence of T2DM $(24,25)$. All 
Table 2 Diagnostic performance of controlled attenuation parameter (CAP) for steatosis grade greater or equal than 1, greater or equal than 2 , and equal to 3

\begin{tabular}{|c|c|c|c|}
\hline Steatosis grade & $S \geq S 1$ ( $\geq 5 \%$ steatosis) & $\mathrm{S} \geq \mathrm{S} 2$ ( $\geq 34 \%$ steatosis) & $S=S 3$ ( $\geq 67 \%$ steatosis) \\
\hline Prevalence (N) & $0.94(\mathrm{~N}=80)$ & $0.65(\mathrm{~N}=55)$ & $0.32(\mathrm{~N}=27)$ \\
\hline \multicolumn{4}{|l|}{ Youden index } \\
\hline Cut-off (dB/m) & 278 & 305 & 307 \\
\hline $\mathrm{TP} /(\mathrm{TP}+\mathrm{FN})$ & $66 / 80$ & $43 / 55$ & $27 / 27$ \\
\hline $\mathrm{Sp}(95 \% \mathrm{Cl})$ & $0.80(0.28-0.99)$ & $0.87(0.69-0.96)$ & $0.71(0.57-0.82)$ \\
\hline $\mathrm{TN} /(\mathrm{TN}+\mathrm{FP})$ & $4 / 5$ & $26 / 30$ & $41 / 58$ \\
\hline PPV (95\% Cl) & $0.99(0.91-1.00)$ & $0.91(0.79-0.97)$ & $0.61(0.46-0.75)$ \\
\hline LR- (95\% Cl) & $0.22(0.12-0.39)$ & $0.25(0.15-0.42)$ & 0 \\
\hline \multicolumn{4}{|l|}{$\mathrm{Se}=0.9$} \\
\hline Cut-off (dB/m) & 270 & 286 & 309 \\
\hline Se $(95 \% \mathrm{Cl})$ & $0.9(0.81-0.96)$ & $0.91(0.80-0.97)$ & $0.96(0.81-1.00)$ \\
\hline $\mathrm{TP} /(\mathrm{TP}+\mathrm{FN})$ & $72 / 80$ & $50 / 55$ & $26 / 27$ \\
\hline $\mathrm{Sp}(95 \% \mathrm{Cl})$ & $0.60(0.15-0.95)$ & $0.63(0.44-0.80)$ & $0.71(0.57-0.82)$ \\
\hline $\mathrm{TN} /(\mathrm{TN}+\mathrm{FP})$ & $3 / 5$ & $19 / 30$ & $41 / 58$ \\
\hline PPV (95\% Cl) & $0.97(0.90-1.00)$ & $0.82(0.70-0.90)$ & $0.60(0.44-0.74)$ \\
\hline $\mathrm{Se}(95 \% \mathrm{Cl})$ & $0.49(0.37-0.60)$ & $0.68(0.54-0.79)$ & $0.52(0.32-0.71)$ \\
\hline $\mathrm{TP} /(\mathrm{TP}+\mathrm{FN})$ & $30 / 80$ & $37 / 55$ & $14 / 27$ \\
\hline $\mathrm{Sp}(95 \% \mathrm{Cl})$ & $1(0.54-1.00)$ & $0.90(0.73-0.98)$ & $0.90(0.79-0.96)$ \\
\hline $\mathrm{TN} /(\mathrm{TN}+\mathrm{FP})$ & $5 / 5$ & $27 / 30$ & $52 / 58$ \\
\hline PPV (95\% Cl) & $1(0.86-1.00)$ & $0.93(0.79-0.98)$ & $0.70(0.46-0.87)$ \\
\hline NPV (95\% Cl) & $0.09(0.03-0.21)$ & $0.60(0.44-0.74)$ & $0.80(0.68-0.89)$ \\
\hline $\mathrm{LR}+(95 \% \mathrm{Cl})$ & - & $6.73(2.26-20.00)$ & $5.01(2.16-11.61)$ \\
\hline LR- (95\% Cl) & $0.63(0.53-0.74)$ & $0.36(0.25-0.54)$ & $0.54(0.36-0.80)$ \\
\hline
\end{tabular}

AUROC, area under the receiver operating curve; $\mathrm{Cl}$, confidence interval; $\mathrm{FN}$, number of false negatives; FP, number of false positive, LR-, negative likelihood ratio; LP+, positive likelihood ratio; NPV, negative predictive value; PPV, positive predictive value; S, steatosis; Se, sensitivity; Sp, specificity; TN, true negative; TP, true positive. 
Table 3 Diagnostic performance of liver stiffness measurement (LSM) for each fibrosis stage grade greater or equal than 2, greater or equal than 3 , and equal to 4

\begin{tabular}{|c|c|c|c|}
\hline Fibrosis stage grade & $F \geq F 2$ & $F \geq F 3$ & $\mathrm{~F}=\mathrm{F} 4$ \\
\hline Prevalence (N) & $0.52(\mathrm{~N}=44)$ & $0.29(\mathrm{~N}=25)$ & $0.08(\mathrm{~N}=7)$ \\
\hline \multicolumn{4}{|l|}{ Youden index } \\
\hline Cut-off (dB/m) & 12.8 & 13.8 & 20.1 \\
\hline $\mathrm{TP} /(\mathrm{TP}+\mathrm{FN})$ & $32 / 44$ & $18 / 25$ & $6 / 7$ \\
\hline Sp $(95 \%$ Cl) & $0.78(0.62-0.89)$ & $0.78(0.65-0.88)$ & $0.90(0.81-0.95)$ \\
\hline $\mathrm{TN} /(\mathrm{TN}+\mathrm{FP})$ & $32 / 41$ & $47 / 60$ & $70 / 78$ \\
\hline PPV (95\% Cl) & $0.77(0.60-0.88)$ & $0.58(0.39-0.75)$ & $0.43(0.19-0.70)$ \\
\hline LR- (95\% Cl) & $0.41(0.26-0.64)$ & $0.36(0.19-0.68)$ & $0.16(0.03-0.98)$ \\
\hline \multicolumn{4}{|l|}{$\mathrm{Se}=0.9$} \\
\hline Cut-off (dB/m) & 8.5 & 10.6 & 14.1 \\
\hline Se $(95 \% \mathrm{Cl})$ & $0.91(0.77-0.97)$ & $0.92(0.72-0.99)$ & $1.00(0.59-1.00)$ \\
\hline $\mathrm{TP} /(\mathrm{TP}+\mathrm{FN})$ & $40 / 44$ & $23 / 25$ & $7 / 7$ \\
\hline $\mathrm{Sp}(95 \% \mathrm{Cl})$ & $0.24(0.13-0.41)$ & $0.45(0.32-0.58)$ & $0.72(0.60-0.81)$ \\
\hline $\mathrm{TN} /(\mathrm{TN}+\mathrm{FP})$ & $10 / 41$ & $27 / 60$ & $56 / 78$ \\
\hline PPV (95\% Cl) & $0.56(0.44-0.68)$ & $0.41(0.28-0.55)$ & $0.24(0.11-0.44)$ \\
\hline $\mathrm{Se}(95 \% \mathrm{Cl})$ & $0.45(0.31-0.61)$ & $0.52(0.32-0.72)$ & $0.86(0.42-0.99)$ \\
\hline $\mathrm{TP} /(\mathrm{TP}+\mathrm{FN})$ & $20 / 44$ & $13 / 25$ & $6 / 7$ \\
\hline $\mathrm{Sp}(95 \% \mathrm{Cl})$ & $0.90(0.76-0.97)$ & $0.90(0.79-0.96)$ & $0.90(0.81-0.95)$ \\
\hline $\mathrm{TN} /(\mathrm{TN}+\mathrm{FP})$ & $37 / 41$ & $54 / 60$ & $70 / 78$ \\
\hline PPV (95\% Cl) & $0.83(0.62-0.95)$ & $0.68(0.43-0.86)$ & $0.43(0.19-0.70)$ \\
\hline NPV (95\% Cl) & $0.61(0.47-0.73)$ & $0.82(0.70-0.90)$ & $0.98(0.91-1.00)$ \\
\hline $\mathrm{LR}+(95 \% \mathrm{Cl})$ & $4.66(1.74-12.48)$ & $5.20(2.23-12.13)$ & $8.36(4.06-17.22)$ \\
\hline LR- (95\% Cl) & $0.60(0.45-0.80)$ & $0.53(0.35-0.80)$ & $0.16(0.03-0.98)$ \\
\hline
\end{tabular}

AUROC, area under the receiver operating curve; $\mathrm{Cl}$, confidence interval; FN, number of false negatives; FP, number of false positive; LR-, negative likelihood ratio; LP+, positive likelihood ratio; NPV, negative predictive value; PPV, positive predictive value; S, steatosis; Se, sensitivity; Sp, specificity; TN, true negative; TP, true positive. 

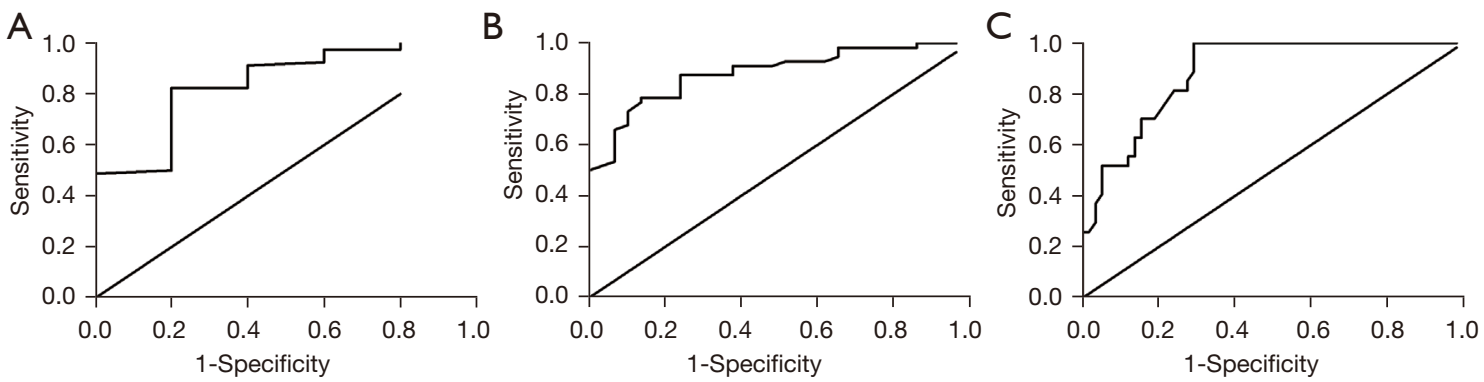

Figure 4 ROC curves for FibroTouch diagnosis of different degrees of liver steatosis. (A) ROC curve for CAP diagnosis of S $\geq$ S1; (B) ROC curve for CAP diagnosis of $\mathrm{S} \geq \mathrm{S} 2$; (C) ROC curve for CAP diagnosis of $\mathrm{S}=\mathrm{S} 3$. CAP, controlled attenuation parameter; ROC, receiver operating characteristic.

A

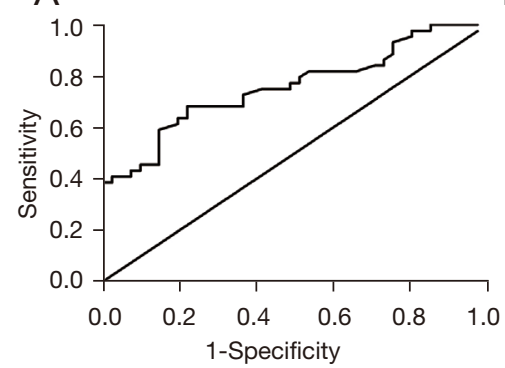

B

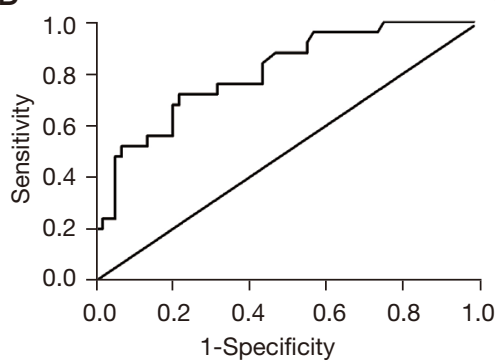

C

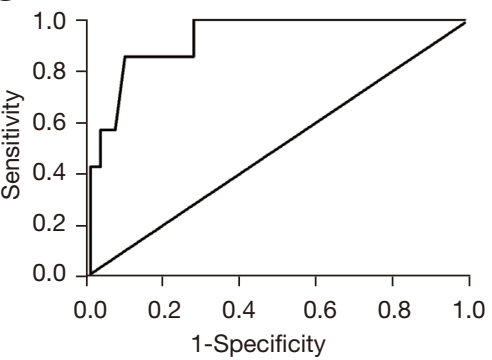

Figure 5 ROC curves for FibroTouch diagnosis of different degrees of liver fibrosis. (A) ROC curve for LSM diagnosis of F $\geq$ F2; (B) ROC curve for LSM diagnosis of F $\geq \mathrm{F} 3$; (C) ROC curve for LSM diagnosis of F = F4. LSM, liver stiffness measurement; ROC, receiver operating characteristic.

subjects included in our current study were patients with T2DM, among whom $94.1 \%$ were also diagnosed with MAFLD, $11.8 \%$ with simple liver steatosis, $82.4 \%$ with steatohepatitis, $40.0 \%$ with fibrotic NASH and $8.2 \%$ with suspected cirrhosis, which were consistent with the reported prevalence rates of steatohepatitis, fibrosis and cirrhosis in patients with T2DM $(5,10,11)$. Progression of MAFLD to advanced liver fibrosis is associated with significantly increased all-cause mortality and liver disease-related mortality (1). Since T2DM can promote the pathogenesis of MAFLD, an assessment of MAFLD severity is required when MAFLD is combined with T2DM. Early diagnosis and treatment will help avoid adverse liver outcomes.

Accurate quantification of liver steatosis and liver fibrosis in patients with MAFLD and monitoring their dynamic changes are important for disease treatment and prognosis. Liver biopsy remains the gold standard for staging liver steatosis and fibrosis. However, its invasiveness leads to poor patient acceptance and limits its clinical application. TE has been widely applied in clinical settings. In particular, the value of FibroScan for assessing the degree of liver fibrosis has been validated in different types of liver disease, and is included in many expert consensuses and guidelines (13). FT integrating 2D imaging and TE techniques use builtin 2D ultrasound to guide accurate localization and detect liver tissue morphology, degree of liver fibrosis and steatosis. FT, as a modified TE technique, can adjust the penetration and resolution according to different examinees, and thus improve the success rate and accuracy of the examination (16). Thus, for obese people, FT has more advantages than FC. Moreover, the FT can automatically adjust the dynamic probes according to the thickness of the subcutaneous fat to increase the rate of reliable measurements and shorten detection time $(16,19)$. It was found that FT had considerable diagnostic efficacy in fibrosis staging in patients with chronic viral hepatitis (26). Also, it had a good correlation with liver histopathology in the staging of liver steatosis and fibrosis $(17,19)$. However, FT has been used in clinical settings for only a short period. However, few studies have investigated FT diagnostic 
thresholds for grading liver steatosis and fibrosis in patients with MAFLD combined with T2DM. In our current prospective study, patients with MAFLD combined with T2DM who underwent liver biopsy and FT examination during the same period were enrolled, and correlations of iver stiffness measurement and controlled attenuation parameter with liver histology were analyzed. As with a previous study (27), there were close relationships between CAP and degree of steatosis, LSM and degree of liver fibrosis. Gender, age and BMI had no effect on LSM and CAP, which was consistent with the results of previous studies (16). Our subjects were 24-74 years old, with a BMI of $22.8-42.3 \mathrm{~kg} / \mathrm{m}^{2}$, and therefore our findings were applicable for most Chinese patients with MAFLD.

This study also provided optimal cut-off values (Youden index criteria, 90\% sensitivity, and $90 \%$ specificity) for FT to stage steatosis and fibrosis in patients with MAFLD combined with T2DM under different clinical scenarios. When the degree of steatosis gradually increased from S0 to $\mathrm{S} 3$ (based on the maximum Youden index), the cutoff values of $C A P$ for $S \geq S 1, S \geq S 2$, and $S=S 3$ were 278,305 , and $307 \mathrm{~dB} / \mathrm{m}$, respectively, with a slight difference in CAP cutoff values between the $\mathrm{S} \geq \mathrm{S} 2$ and $\mathrm{S}=\mathrm{S} 3$. In clinical practice, identifying the moderate to severe steatosis is more valuable than distinguishing S2 from S3. Therefore, the cutoff value of CAP set at $305 \mathrm{~dB} / \mathrm{m}$ for the diagnosis of $\mathrm{S} \geq$ $\mathrm{S} 2$ is of great significance for clinical work. The AUROCs of CAP detection using FT for $\mathrm{S} \geq \mathrm{S} 2$ and $\mathrm{S}=\mathrm{S} 3$ were 0.88 (0.81-0.95) and 0.89 (0.82-0.95), where they were higher than those of FibroScan [AUROC 0.77 (0.71-0.82), 0.70 $(0.64-0.75)]$, respectively (28). This is due to a broadband probe that emits a wider ultrasound band $(2-7 \mathrm{MHz})$ with both high-frequency and low-frequency components, which can adjust the penetration and resolution according to different examinees. Thus, FT is especially valuable for obese people, and its efficacy in staging steatosis and fibrosis in patients with nonalcoholic fatty liver.

Liver fibrosis is an important prognostic factor for chronic liver disease $(29,30)$, and proper assessment and timely detection of advanced liver fibrosis and early cirrhosis are key steps in optimizing the management of chronic liver disease. This study found that LSM detected by FT in patients with MAFLD combined with T2DM was closely related to the histological stage of liver fibrosis, and there were clear cutoff values between different degrees of liver fibrosis. LSM increased gradually from F0 to F4. Since there was no statistical difference in LSM between $\mathrm{F} 0$ and $\mathrm{F} 1$ groups, and $\mathrm{P}=0.117$ for the $\mathrm{F} \geq \mathrm{F} 1 \mathrm{ROC}$ curve, only the ROC curves were constructed for $\mathrm{F} \geq \mathrm{F} 2, \mathrm{~F} \geq$ $\mathrm{F} 3$, and $\mathrm{F}=\mathrm{F} 4$. LSM cutoff values were $12.8,13.8$, and $20.1 \mathrm{kPa}$, respectively, based on the corresponding Youden index, which was higher than the cutoff values of FT in the diagnosis of liver fibrosis in patients with chronic viral hepatitis B (19), which could be explained by the different pathogenic mechanisms of these two diseases. Liver steatosis is negatively associated with CHB infection in some reports (31), but when patients coexisting CHB and MAFLD, liver steatosis may accelerate the progression of liver disease in patients with CHB. Liver steatosis and chronic hepatitis B can synergistically induce liver cirrhosis or even HCC development (32). LSM had a good NPV (0.87) in the diagnosis of $\mathrm{F} \geq \mathrm{F} 3$; in addition, LSM had high sensitivity (0.86) and specificity (0.90) and a good NPV (0.99) in the diagnosis of $\mathrm{F}=\mathrm{F} 4$. Pairwise analysis revealed no statistical difference in LSM between the F1 and F2 groups. Therefore, detection of LSM with FT may be more beneficial in distinguishing between liver fibrosis without/ without fibrosis (F0/F1) and advanced liver fibrosis (F $\geq F 3$ ).

Due to its prospective diagnostic design, our study provided the cutoff values for FT to stage liver steatosis and fibrosis in different clinical situations, and could help clinicians assess disease conditions in MAFLD patients with T2DM. However, its small sample size limited our study and single-center design, we are still carrying out relevant research based on this research, and we look forward to a larger sample study for verification in the future. Furthermore, steatosis was not found in some biopsy samples, which could be related to the uneven distribution of adipose tissue and the technical limitations of liver tissue puncture, although these are also the real-world characteristics of this technique.

In conclusion, LSM and CAP detected by FT in MAFLD patients with T2DM are closely correlated with liver fibrosis and steatosis stages confirmed by liver biopsy, showing high diagnostic efficacy. In addition, our study provided a comprehensive range of LSM and CAP cutoff values, which provides a reliable basis for the application of FT in T2DM patients.

\section{Acknowledgments}

Funding: Tianjin Science and technology major project of chronic disease prevention and control (17ZXMFSY00170); Tianjin Key projects of health and family planning commission (15-KG115); Tianjin research program of application foundation and advanced technology 
(15JCYBJC27700).

\section{Footnote}

Reporting Checklist: The authors have completed the STARD reporting checklist. Available at https://dx.doi. org/10.21037/apm-21-2339

Data Sharing Statement: Available at https://dx.doi. org/10.21037/apm-21-2339

Conflicts of Interest: All authors have completed the ICMJE uniform disclosure form (available at https://dx.doi. org/10.21037/apm-21-2339). The authors have no conflicts of interest to declare.

Ethical Statement: The authors are accountable for all aspects of the work in ensuring that questions related to the accuracy or integrity of any part of the work are appropriately investigated and resolved. The Ethics Committee of the Third Central Hospital of Tianjin approved this study (IRB2016-006-02). This study was conducted in accordance with the Declaration of Helsinki (as revised in 2013) and Good Clinical Practice (GCP) for drugs. All subjects signed informed consents.

Open Access Statement: This is an Open Access article distributed in accordance with the Creative Commons Attribution-NonCommercial-NoDerivs 4.0 International License (CC BY-NC-ND 4.0), which permits the noncommercial replication and distribution of the article with the strict proviso that no changes or edits are made and the original work is properly cited (including links to both the formal publication through the relevant DOI and the license). See: https://creativecommons.org/licenses/by-nc-nd/4.0/.

\section{References}

1. Cotter TG, Rinella M. Nonalcoholic Fatty Liver Disease 2020: The State of the Disease. Gastroenterology 2020;158:1851-64.

2. Eslam M, Newsome PN, Sarin SK, et al. A new definition for metabolic dysfunction-associated fatty liver disease: An international expert consensus statement. J Hepatol 2020;73:202-9.

3. Sharma P, Arora A. Clinical presentation of alcoholic liver disease and non-alcoholic fatty liver disease: spectrum and diagnosis. Transl Gastroenterol Hepatol 2020;5:19.
4. Angulo P. Nonalcoholic fatty liver disease. N Engl J Med 2002;346:1221-31.

5. Younossi ZM, Koenig AB, Abdelatif D, et al. Global epidemiology of nonalcoholic fatty liver disease-Metaanalytic assessment of prevalence, incidence, and outcomes. Hepatology 2016;64:73-84.

6. Younossi Z, Tacke F, Arrese M, et al. Global Perspectives on Nonalcoholic Fatty Liver Disease and Nonalcoholic Steatohepatitis. Hepatology 2019;69:2672-82.

7. Loomba R, Abraham M, Unalp A, et al. Association between diabetes, family history of diabetes, and risk of nonalcoholic steatohepatitis and fibrosis. Hepatology 2012;56:943-51.

8. Lee YH, Cho Y, Lee BW, et al. Nonalcoholic Fatty Liver Disease in Diabetes. Part I: Epidemiology and Diagnosis. Diabetes Metab J 2019;43:31-45.

9. Khneizer G, Rizvi S, Gawrieh S. Non-alcoholic Fatty Liver Disease and Diabetes Mellitus. Adv Exp Med Biol 2021;1307:417-40.

10. Williams CD, Stengel J, Asike MI, et al. Prevalence of nonalcoholic fatty liver disease and nonalcoholic steatohepatitis among a largely middle-aged population utilizing ultrasound and liver biopsy: a prospective study. Gastroenterology 2011;140:124-31.

11. Younossi ZM, Marchesini G, Pinto-Cortez H, et al. Epidemiology of Nonalcoholic Fatty Liver Disease and Nonalcoholic Steatohepatitis: Implications for Liver Transplantation. Transplantation 2019;103:22-7.

12. Ekstedt M, Hagström H, Nasr P, et al. Fibrosis stage is the strongest predictor for disease-specific mortality in NAFLD after up to 33 years of follow-up. Hepatology 2015;61:1547-54.

13. Kemp W, Levy M, Weltman M, et al. Australian Liver Association (ALA) expert consensus recommendations for the use of transient elastography in chronic viral hepatitis. J Gastroenterol Hepatol 2015;30:453-62.

14. Lee BW, Lee YH, Park CY, et al. Non-Alcoholic Fatty Liver Disease in Patients with Type 2 Diabetes Mellitus: A Position Statement of the Fatty Liver Research Group of the Korean Diabetes Association. Diabetes Metab J 2020;44:382-401.

15. Singh S, Muir AJ, Dieterich DT, et al. American Gastroenterological Association Institute Technical Review on the Role of Elastography in Chronic Liver Diseases. Gastroenterology 2017;152:1544-77.

16. Zeng J, Sun WL, Chen GY, et al. Efficiency of FibroScan and FibroTouch in liver stiffness measurement and fat quantification: a comparative analysis. Zhonghua Gan 
Zang Bing Za Zhi 2016;24:652-8.

17. Zhu SH, Zheng KI, Hu DS, et al. Optimal thresholds for ultrasound attenuation parameter in the evaluation of hepatic steatosis severity: evidence from a cohort of patients with biopsy-proven fatty liver disease. Eur J Gastroenterol Hepatol 2021;33:430-5.

18. Yang XZ, Gen AW, Xian JC, et al. Diagnostic value of various noninvasive indexes in the diagnosis of chronic hepatic fibrosis. Eur Rev Med Pharmacol Sci 2018;22:479-85.

19. Xu Y, Liu Y, Cao Z, et al. Comparison of FibroTouch and FibroScan for staging fibrosis in chronic liver disease: Single-center prospective study. Dig Liver Dis 2019;51:1323-9.

20. The Chinese National Workshop on Fatty Liver and Alcoholic Liver Disease for the Chinese Liver Disease Association. Guidelines for management of nonalcoholic fatty liver disease: an updated and revised edition. Chin J Hepatol 2010;18:163-6.

21. European Association for the Study of the Liver (EASL); European Association for the Study of Diabetes (EASD); European Association for the Study of Obesity (EASO). EASL-EASD-EASO Clinical Practice Guidelines for the management of non-alcoholic fatty liver disease. J Hepatol 2016;64:1388-402.

22. Kleiner DE, Brunt EM, Van Natta M, et al. Design and validation of a histological scoring system for nonalcoholic fatty liver disease. Hepatology 2005;41:1313-21.

23. Eslam M, Sanyal AJ, George J, et al. MAFLD: A Consensus-Driven Proposed Nomenclature for Metabolic Associated Fatty Liver Disease. Gastroenterology 2020;158:1999-2014.e1.

24. Angulo P, Keach JC, Batts KP, et al. Independent predictors of liver fibrosis in patients with nonalcoholic

Cite this article as: Yu H, Liu H, Zhang J, Jia G, Yang L, Zhang Q, Li G, Liu F, Di F, Wang F. Accuracy of FibroTouch in assessing liver steatosis and fibrosis in patients with metabolicassociated fatty liver disease combined with type 2 diabetes mellitus. Ann Palliat Med 2021;10(9):9702-9714. doi: 10.21037/ apm-21-2339 steatohepatitis. Hepatology 1999;30:1356-62.

25. Leite NC, Villela-Nogueira CA, Pannain VL, et al. Histopathological stages of nonalcoholic fatty liver disease in type 2 diabetes: prevalences and correlated factors. Liver Int 2011;31:700-6.

26. Duan WJ, Wang XZ, Ma AL, et al. Multicenter prospective study to validate a new transient elastography device for staging liver fibrosis in patients with chronic hepatitis B. J Dig Dis 2020;21:519-25.

27. Shen F, Zheng RD, Mi YQ, et al. Controlled attenuation parameter for non-invasive assessment of hepatic steatosis in Chinese patients. World J Gastroenterol 2014;20:4702-11.

28. Eddowes PJ, Sasso M, Allison M, et al. Accuracy of FibroScan Controlled Attenuation Parameter and Liver Stiffness Measurement in Assessing Steatosis and Fibrosis in Patients With Nonalcoholic Fatty Liver Disease. Gastroenterology 2019;156:1717-30.

29. Dulai PS, Singh S, Patel J, et al. Increased risk of mortality by fibrosis stage in nonalcoholic fatty liver disease: Systematic review and meta-analysis. Hepatology 2017;65:1557-65.

30. Méndez-Sánchez N, Valencia-Rodríguez A, CoronelCastillo C, et al. The cellular pathways of liver fibrosis in non-alcoholic steatohepatitis. Ann Transl Med 2020;8:400.

31. Joo EJ, Chang Y, Yeom JS, et al. Hepatitis B virus infection and decreased risk of nonalcoholic fatty liver disease: A cohort study. Hepatology 2017;65:828-35.

32. Choi HSJ, Brouwer WP, Zanjir WMR, et al. Nonalcoholic Steatohepatitis Is Associated With Liver-Related Outcomes and All-Cause Mortality in Chronic Hepatitis B. Hepatology 2020;71:539-48.

(English Language Editor: J. Chapnick) 